Short Communication

\title{
Application of High-Speed Countercurrent Chromatography to the Separation of Flavanonol, Phenylcoumaran and Flavonolignans in Silybum marianum
}

\author{
Kazumasa ZAIMA ${ }^{1}$, Risako KOGA ${ }^{1}$, Riku MOTEGI ${ }^{1}$, Hanae SATO $^{1}$, Susumu KITANAKA ${ }^{1}$, \\ Yoichiro ITO², Kazufusa SHINOMIYA*1 \\ ${ }^{1}$ School of Pharmacy, Nihon University, 7-7-1, Narashinodai, Funabashi 274-8555, Japan \\ ${ }^{2}$ Laboratory of Bioseparation Technology, Biochemistry and Biophysics Center, Division of Intramural Research, National
}

Heart, Lung, and Blood Institute, National Institutes of Health, 10 Center Drive, MSC 1762, Building 10, Room 5D18,

Bethesda, MD 20892-1762, USA

\begin{abstract}
Separation of flavanonol, phenylcoumaran and flavonolignans in Silybum marianum was examined using high-speed countercurrent chromatography (HSCCC). In order to prepare analytical standards, a flavanonol of aromadendrin $(87.4 \%$ purity, $7.8 \mathrm{mg})$, three phenylcoumarans of jatrointelignan D $(84.5 \%$ purity, $13.2 \mathrm{mg})$, dehydrodiconiferyl alcohol $(76.1 \%$ purity, $1.4 \mathrm{mg}$ ) and dihydrodehydrodiconiferyl alcohol $(93.0 \%$ purity, $5.8 \mathrm{mg})$, and three flavonolignans of silybin ( $88.8 \%$ purity, 35.6 $\mathrm{mg}$ ), silydianin ( $99.3 \%$ purity, $25.1 \mathrm{mg})$ and silychristin $(96.9 \%$ purity, $12.6 \mathrm{mg}$ ) were separated from the seeds of S. marianum using common column chromatography and ODS-HPLC, and identified by ${ }^{1} \mathrm{H}$ and ${ }^{13} \mathrm{C}$ NMR spectra. Then, HSCCC with the hexane/ethyl acetate/methanol/water $(3: 7: 4: 6, \mathrm{v} / \mathrm{v})$ system was applied to the separation of aromadendrin, jatrointelignan $\mathrm{D}$, silydianin and silybin. In this separation, it was revealed that silybin and silydianin were successfully separated from each other. The present HSCCC system was directly applied to the ethyl acetate extract and resulted in the separation of silybin. The overall results suggested that HSCCC is useful for the separation of bioactive compounds in S. marianum.
\end{abstract}

Keywords: Separation; High-speed countercurrent chromatography; Silybum marianum; Natural products

\section{Introduction}

The genus Silybum is a member of the Asteraceae family and Silybum spp. are widely distributed in Mediterranean region, North Africa, Asia and also in Japan. The crude extract of $S$. marianum is used for apepsy and the further purified product is used for chronic hepatitis and cirrhosis of liver in Germany. It is known that $S$. marianum contains many flavonolignans, sterols and flavonoids [1-4]. In particular, silymarin is contained at about $30-65 \%$ in the seeds of $S$. marianum and it is composed of silybin, isosilybin A, isosilybin B, silychristin, isosilychristin, silydianin and taxifolin [5-7]. Silymarin has antioxidant activity, antifibrotic activity, hepatic protection effect, etc., [5,7-10] where silybin covers approximately $50-70 \%$ of silymarin with other components including isosilybin

${ }^{*}$ Corresponding author: Kazufusa SHINOMIYA

Tel: +81-47-465-6137; Fax: +81-47-465-2158

E-mail: shinomiya.kazufusa@nihon-u.ac.jp (about 5\%), silychristin (about 20\%) and silydianin (about $10 \%$ ) as well as silimonin, isosilychristin, isosilibinin, etc [7]. Several biological activities of silymarin as described above may be caused by silybin. From this reason, simple separation of the bioactive compounds in $S$. marianum is required to obtain at the preparative level without loss of samples.

Countercurrent chromatography (CCC) is a support-free chromatography based on the liquid-liquid partitioning of analytes between two immiscible liquid phases in the column. This distinctive feature eliminates the adsorption and the denaturation of the target compound caused by the interaction between the solutes and the solid stationary phase which is sometimes observed in the use of common column chromatography. $\mathrm{CCC}$ has been widely used for

Received: 30 January 2020

Accepted: 12 April 2020

J-STAGE Advance Published: 24 April 2020

DOI: $10.15583 /$ jpchrom.2020.006 
the separation and the purification of various bioactive compounds in the natural products [11-13]. Among many CCC instruments developed in the past, the high-speed CCC (HSCCC) is useful for effective separation of natural compounds within shorter separation times.

This paper describes the application of HSCCC to the separation of flavanonol, phenylcoumaran and flavonolignans in S. marianum.

\section{Experimental}

\subsection{Apparatus}

The HSCCC apparatus employed in the present study was constructed at the Machining Technology Center of Nihon University, Chiba, Japan. The design and the fabrication of the apparatus have been described in elsewhere [14,15]. For each separation, four eccentric coil assemblies were equipped in rotary frame of the HSCCC apparatus. Each eccentric coil assembly was prepared by winding $1 \mathrm{~mm}$ I.D. PTFE (polytetrafluoroethylene) tubing (Flon Industry, Tokyo, Japan) onto $5 \mathrm{~cm}$ long, $5 \mathrm{~mm}$ O.D. nylon pipes making a series of tight left-handed coils for the forward type-J planetary motion or right-handed coils for the backward type-I planetary motion (20 turns for 1 unit). These coil units were arranged symmetrically around the holder hub of $5 \mathrm{~cm}$ O.D. in such a way that the axis of each coil unit is parallel to the holder axis (13 units for the first layer and 20 units for the second layer). The total capacity of four columns connected in series was $55 \mathrm{~mL}$.

The HPLC equipment consists of a reciprocating pump (Type LC-20AD, Shimadzu Corporation, Kyoto, Japan), a UV detector (Type SPD-10A $A_{\mathrm{VP}}$, Shimadzu), a separation column (Inertsil ODS-4, $250 \times 4.6 \mathrm{~mm}$ I.D., $5 \mu \mathrm{m}$, GL Sciences, Tokyo, Japan for the analytical scale, Inertsil ODS-4, $250 \times 14 \mathrm{~mm}$ I.D., $5 \mu \mathrm{m}$, GL Sciences and COSMOSIL Cholester, $250 \times 10 \mathrm{~mm}$ I.D., $5 \mu \mathrm{m}$, Nacalai tesque, Kyoto, Japan for the preparative scale).

${ }^{1} \mathrm{H},{ }^{13} \mathrm{C}$ and $2 \mathrm{D}$ NMR spectra were measured using a spectrometer (Type ECA-500, JEOL, Tokyo, Japan) and the chemical shifts were referenced to the residual solvent peaks of methanol- $d_{4}\left(\mathrm{CD}_{3} \mathrm{OD}\right)$ or acetone- $d_{6}\left(\left(\mathrm{CD}_{3}\right)_{2} \mathrm{CO}\right)$ as an internal standard $\left(\delta_{\mathrm{H}} 3.31\right.$ and $\delta_{\mathrm{C}} 49.0$ for $\mathrm{CD}_{3} \mathrm{OD}$, or $\delta_{\mathrm{H}}$ 2.05 and $\delta_{\mathrm{C}} 29.84$ for $\left(\left(\mathrm{CD}_{3}\right)_{2} \mathrm{CO}\right)$.

\subsection{Reagents and plant material}

Hexane, ethyl acetate (EtOAc), methanol $(\mathrm{MeOH})$ and 1-butanol were of the first grade purchased from FUJIFILM Wako Pure Chemical Corporation (Osaka, Japan). All other reagents were of reagent grade. The seeds of $S$. marianum were obtained from a market at Hebei in China.

\subsection{Separation and identification of flavanonol,} phenylcoumarans and flavonolignans in S. marianum

The seeds of $S$. marianum $(1.5 \mathrm{~kg})$ were extracted with
$\mathrm{MeOH}$, and a part of the extract ( $36.8 \mathrm{~g}$ ) was dissolved with water and then partitioned with hexane. The aqueous layer was further partitioned with EtOAc to give the extract $(25.6 \mathrm{~g})$. A part of this extract $(3.6 \mathrm{~g})$ was subjected to a silica gel column (chloroform $\left(\mathrm{CHCl}_{3}\right) / \mathrm{MeOH}, 1: 0 \rightarrow 50$ : $1 \rightarrow 19: 1 \rightarrow 9: 1 \rightarrow 8: 2 \rightarrow 1: 1 \rightarrow 0: 1)$ to give 13 fractions. The fraction eluted with $\mathrm{CHCl}_{3} / \mathrm{MeOH}(50: 1)$ was subjected to passage over an ODS column (water/MeOH, $6: 4 \rightarrow 1: 1 \rightarrow 4: 6 \rightarrow 3: 7 \rightarrow 2: 8 \rightarrow 0$ : 1) to give jatrointelignan $D[16]$ and silybin [2]. The fraction eluted with $\mathrm{CHCl}_{3} / \mathrm{MeOH}(9: 1)$ was subjected to separate over an ODS column (water/MeOH, $8: 2 \rightarrow 6: 4$ $\rightarrow 1: 1 \rightarrow 4: 6 \rightarrow 2: 8 \rightarrow 0: 1)$ followed by ODS-HPLC (acetonitrile/ $0.1 \%$ formic acid, $3: 7$ ) using COSMOSIL Cholester column to give dehydrodiconiferyl alcohol [17,18], dihydrodehydrodiconiferyl alcohol [18] and aromadendrin [19]. Other fractions eluted with water/MeOH $(1: 1)$ were further subjected to an ODS HPLC using Inertsil ODS-4 column and a silica gel column (benzene/EtOAc, $1: 0 \rightarrow 8: 2 \rightarrow 7: 3 \rightarrow 6: 4 \rightarrow 1: 1$ ) to give silydianin [2]. The fraction eluted with $\mathrm{CHCl}_{3} / \mathrm{MeOH}(1: 1)$ was identified as silychristin [2].

\subsection{Measurement of the partition coefficient of analytical standards in the hexane/EtOAc/MeOH/water (HEMWat) system}

In order to select a suitable two-phase solvent system for the HSCCC separation, the partition coefficient $\left(K_{\mathrm{D}}\right)$ values of jatrointelignan $\mathrm{D}$, silybin, aromadendrin and silydianin were measured using the simple test tube method using the hexane/EtOAc/MeOH/water (HEMWat) system established by Friesen et al. [20] as follows: Two milliliters of each phase were put into a test tube, and then about $1 \mathrm{mg}$ of test sample was added and mixed vigorously. After two layers were formed, $1 \mathrm{~mL}$ of each phase was put into another test tube and diluted with $2 \mathrm{~mL}$ of $\mathrm{MeOH}$. The absorbance of this solution was measured at $288 \mathrm{~nm}$ using a spectrophotometer (Type UV-1800, Shimadzu). The wavelength at $288 \mathrm{~nm}$ selected in the present study can commonly detect flavanonols, phenylcoumarans and flavonolignans. The $K_{\mathrm{D}}$ value was calculated from the absorbance of the upper phase divided by that of the lower phase.

\subsection{Preparation of the two-phase solvent system and the sample solution}

Each HEMWat system examined in the present system was prepared at a desired volume ratio as summarized in Table 3. The solvent mixture was stirred vigorously and then settled to form two layers in a separatory funnel at room temperature. The sample solution was prepared by dissolving an aliquot of the sample in equal volume of each phase for HSCCC separation. 


\subsection{HSCCC separation and HPLC analysis of the fraction}

The coiled column was filled with the stationary phase and followed the injection of the sample solution through the column inlet by the syringe. When the rotation speed of the column reached $1000 \mathrm{rpm}$, the mobile phase was pumped into the column at a flow rate of $0.5 \mathrm{~mL} / \mathrm{min}$. The effluent was collected into test tubes at $2 \mathrm{~min} /$ tube using the fraction collector (Advantec. Co., Tokyo, Japan) and measured the absorbance at $288 \mathrm{~nm}$, and then applied to the HPLC analysis. The HPLC conditions are as follows: column: Inertsil ODS-4 $(250 \times 4.6 \mathrm{~mm}$ I.D. $)$, eluent: $0.1 \%$ acetic acid (A), acetonitrile (B), $\mathrm{A} / \mathrm{B}=75: 25$ (initial) $75: 25$ (15 min) - $60: 40$ (10 min) - $60: 40$ (10 $\mathrm{min})$, flow rate: $1.0 \mathrm{~mL} / \mathrm{min}$, column temperature: $40{ }^{\circ} \mathrm{C}$, detection: $288 \mathrm{~nm}$.

\section{Results and discussion}

3.1. Separation and identification of flavanonol, three phenylcoumarans and three flavonolignans

In order to prepare analytical standards, flavanonol, three phenylcoumarans and three flavonolignans were separated from the seeds of $S$. marianum and identified by ${ }^{1} \mathrm{H}$ and ${ }^{13} \mathrm{C}$ NMR spectra. As a result, aromadendrin (87.4\% purity, $7.8 \mathrm{mg})$, jatrointelignan D $(84.5 \%$ purity, $13.2 \mathrm{mg})$, dehydrodiconiferyl alcohol (76.1\% purity, $1.4 \mathrm{mg})$, dihydrodehydrodiconiferyl alcohol (93.0\% purity, $5.8 \mathrm{mg})$, silybin (88.8\% purity, $35.6 \mathrm{mg})$, silydianin (99.3\% purity, $25.1 \mathrm{mg}$ ) and silychristin (96.9\% purity, $12.6 \mathrm{mg}$ ) were obtained from the seeds of $S$. marianum, where the purity of
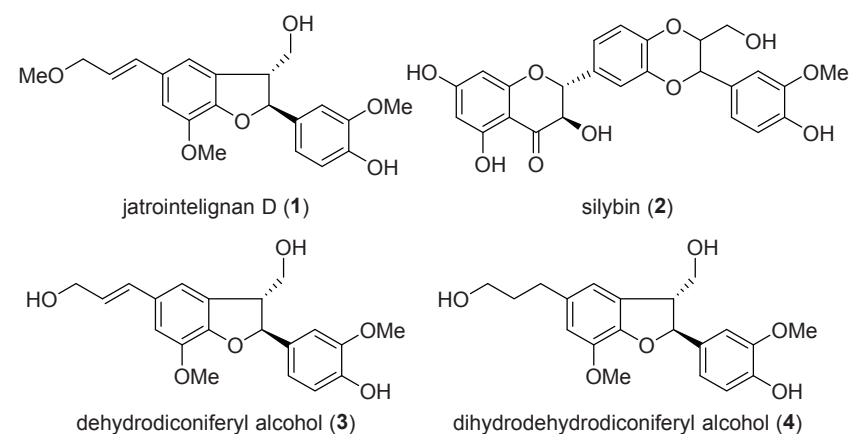

dehydrodiconiferyl alcohol (3)
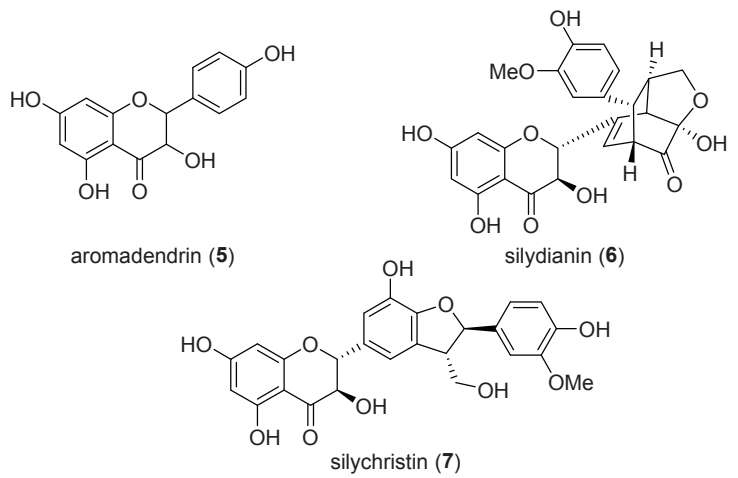

Fig. 1. Chemical structures isolated from the seeds of $S$ marianum. each compound was calculated from the peak area ratio in the HPLC chromatogram. Each spectrophotometric data was corresponded to the previously published data [2,16-19]. The chemical structures are illustrated in Fig. 1.

\subsection{Measurement of the partition coefficient $\left(K_{D}\right)$ and HSCCC separation of the components}

In order to find a suitable two-phase solvent system for HSCCC separation, the $K_{\mathrm{D}}$ values were measured using the simple test tube method for jatrointelignan D, silybin, aromadendrin and silydianin, which were obtained at relatively large amounts. Table 1 summarizes the $K_{\mathrm{D}}$ values in the HEMWat system at various composition ratios. The $K_{\mathrm{D}}$ values of these four compounds were increased according to the increase of the polarity of the two-phase solvent system (the increase of the number of the HEMWat system). In the most hydrophobic composition ratio of the present solvent system (HEMWat -8), all four compounds were almost partitioned into the lower phase, while in the most hydrophilic composition ratio of the present solvent system (HEMWat +8), these compounds were almost partitioned into the upper phase. As the result, the HEMWat +4 system, hexane/EtOAc/MeOH/water (3 : 7 : $4: 6, \mathrm{v} / \mathrm{v}$ ) was selected for the HSCCC separation because the $K_{\mathrm{D}}$ values of these compounds were relatively different from each other.

\subsection{HSCCC separation of components obtained from the seeds of $S$. marianum}

Figure 2 illustrates the HSCCC separation of silydianin, jatrointelignan $\mathrm{D}$, aromadendrin and silybin obtained using the HEMWat system $(3: 7: 4: 6, \mathrm{v} / \mathrm{v})$ with the lower mobile phase. The stationary phase retention was $14.5 \%$, which was extremely low but almost similar elution pattern was obtained using another type of the HSCCC, the small-scale cross-axis CCC, with the stationary phase retention at $41.2 \%$.

As illustrated in Fig. 2, the eluate obtained in this HSCCC separation was divided into four fractions (Fr. a d). The purity of the component containing each fraction was calculated from the HPLC data. Figure 3 shows the HPLC chromatograms of each fraction. In the HSCCC, each compound was separated insufficiently except for silydianin in Fr. b. Fraction c contained both jatrointelignan D and aromadendrin as illustrated in Fig. 3C. However, in Fr. d, silybin was detected as highly purified single peak in the HPLC chromatogram. From these results, it was suggested that silydianin and silybin could be separated in the present HSCCC conditions. When using the HEMWat +5 system, hexane/EtOAc/MeOH/water (3 : $7: 3: 7, \mathrm{v} / \mathrm{v})$, silydianin and jatrointelignan $\mathrm{D}$ may be separated with the lower mobile phase, and silybin and aromadendrin may be separated with the upper mobile 
phase. HSCCC is useful for the preparation of bioactive compounds, because the single HSCCC peak with UV detection is sometimes composed of several constituents at high purities, which is different from HPLC.

Table 1. Composition ratios of the HEMWat system and the partition coefficient $\left(K_{\mathrm{D}}\right)$ values of jatrointelignan D (1), silybin (2), aromadendrin (5) and silydianin (6).

\begin{tabular}{cccccccccc}
\hline \multirow{2}{*}{ HEMWat } & hexane & EtOAc & MeOH & water & \multicolumn{7}{c}{$K_{\mathrm{D}}$} \\
\hline & & & & & $\mathbf{1}$ & $\mathbf{2}$ & $\mathbf{5}$ & $\mathbf{6}$ \\
\hline-8 & 10 & 0 & 10 & 0 & 0.54 & 0.11 & 0.04 & 0.22 \\
-6 & 9 & 1 & 9 & 1 & 0.49 & 0.10 & 0.08 & 0.04 \\
-5 & 8 & 2 & 8 & 2 & 0.44 & 0.03 & 0.01 & 0.01 \\
-4 & 7 & 3 & 7 & 3 & 0.43 & 0.25 & 0.02 & 0.06 \\
-3 & 6 & 3 & 6 & 4 & 0.40 & 0.01 & 0.09 & 0.04 \\
-2 & 7 & 3 & 5 & 5 & 0.27 & 0.11 & 0.02 & 0.08 \\
-1 & 6 & 4 & 5 & 5 & 0.37 & 0.08 & 0.09 & 0.15 \\
0 & 5 & 5 & 5 & 5 & 0.49 & 0.11 & 0.10 & 0.07 \\
+1 & 4 & 6 & 5 & 5 & 0.57 & 0.27 & 0.29 & 0.09 \\
+2 & 3 & 7 & 5 & 5 & 0.66 & 0.56 & 0.59 & 0.15 \\
+3 & 4 & 6 & 4 & 6 & 0.79 & 0.90 & 0.66 & 0.20 \\
+4 & 3 & 7 & 4 & 6 & 1.07 & 2.50 & 1.59 & 0.36 \\
+5 & 3 & 7 & 3 & 7 & 1.76 & 6.63 & 3.47 & 0.48 \\
+6 & 2 & 8 & 2 & 8 & 3.46 & 13.14 & 11.13 & 0.96 \\
+7 & 1 & 9 & 1 & 9 & 6.18 & 14.97 & 16.53 & 1.50 \\
+8 & 0 & 10 & 0 & 10 & 24.19 & 43.49 & 24.17 & 2.01 \\
\hline
\end{tabular}

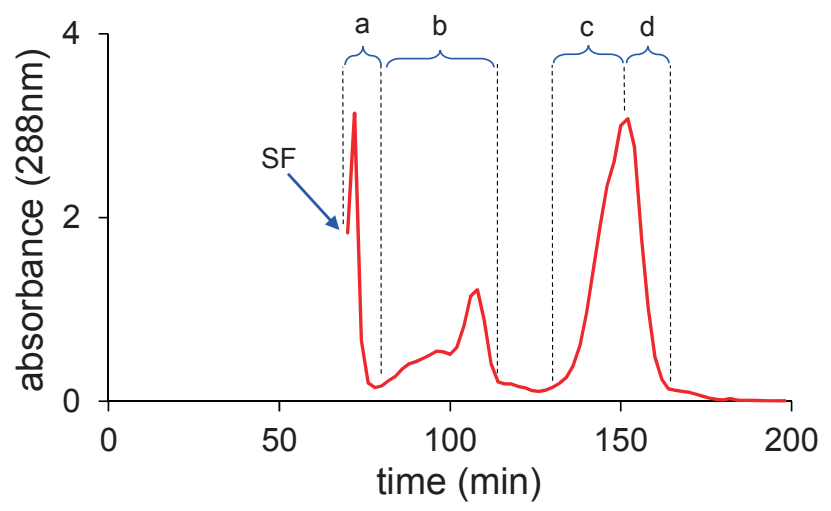

Fig. 2. The elution curve of selected compounds isolated from $S$. marianum using HSCCC. Experimental conditions: Apparatus: the universal HSCCC equipped with eccentric coil assemblies, 1.5 mm I.D. $\times 2.5 \mathrm{~mm}$ O.D., $55 \mathrm{~mL}$ capacity; Sample: jatrointelignan D (1 mg), silybin (1 mg), aromadendrin (1 mg) and silydianin (1 $\mathrm{mg}$ ) for lower mobile phase; Solvent system: HEMWat (3:7:4:6); Rotation speed: $1000 \mathrm{rpm}$; Flow rate: $0.5 \mathrm{~mL} / \mathrm{min}$; Fractionation: $1.0 \mathrm{~mL} /$ tube, Detection: $288 \mathrm{~nm}$; SF: solvent front.

Figure 4 illustrates the HSCCC separation of the EtOAc extract obtained from the seeds of $S$. marianum using the HEMWat $(3: 7: 4: 6, \mathrm{v} / \mathrm{v})$ system with the lower mobile phase. The eluate was divided into 10 fractions. As illustrated in Fig. 5, silybin was successfully separated in Fr. 9 at high purity while the elution time was slightly shorter than that in Fig. 3. This may be caused by lower stationary phase retention at $12.7 \%$ due to the matrix effect of the sample. However, this result suggests that silybin can be directly separated from the EtOAc extract without any purification process.
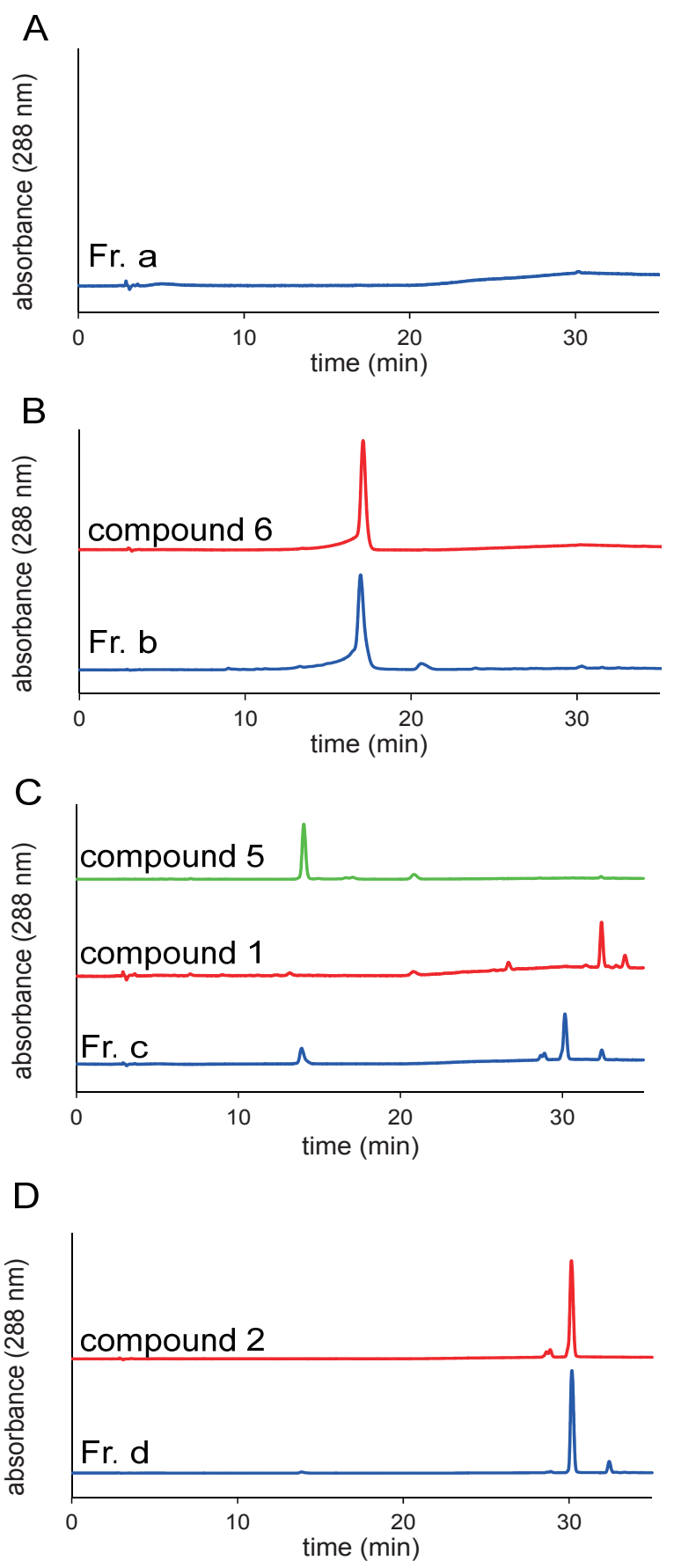

Fig. 3. HPLC chromatograms of standards (red or green) and each fraction (blue) after HSCCC separation. (A) Fraction a, (B) Fraction b and silydianin (6), (C) Fraction c, jatrointelignan D (1) and aromadendrin (5), (D) Fraction $\mathrm{d}$ and silybin (2). Experimental conditions: Column: Inertsil ODS-4 $(4.6 \times 250 \mathrm{~mm}$, $5 \mu \mathrm{m})$; Mobile phase: A/B $0.1 \%$ formic acid/acetonitrile $=75: 25$ (initial) $-75: 25(15 \mathrm{~min})-60: 40(10 \mathrm{~min})-60: 40(10 \mathrm{~min})$; Flow rate: $1.0 \mathrm{~mL} / \mathrm{min}$; Column temperature: $40^{\circ} \mathrm{C}$; Detection: $288 \mathrm{~nm}$. 


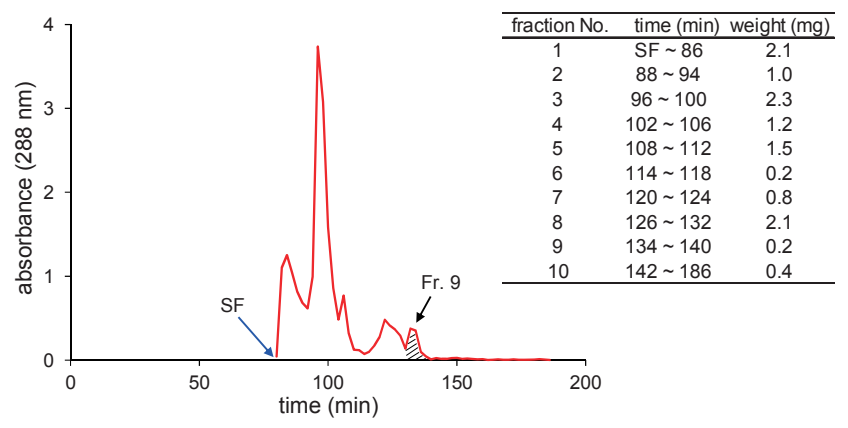

Fig. 4. The elution curve of EtOAc layer extracted from the seeds of $S$. marianum using established HSCCC separation method, and fraction number and elution time of each fraction after HSCCC separation. Experimental conditions: Apparatus: the universal HSCCC equipped with eccentric coil assemblies, 1.5 mm I.D. $\times 2.5 \mathrm{~mm}$ O.D., $55 \mathrm{~mL}$ capacity; Sample: EtOAc layer extracted from the seeds of $S$. marianum for lower mobile phase; Solvent system: HEMWat (3: $7: 4: 6$ ); Rotation speed: 1000 $\mathrm{rpm}$; Flow rate: $0.5 \mathrm{~mL} / \mathrm{min}$; Fractionation: $1.0 \mathrm{~mL} / \mathrm{tube}$, Detection: $288 \mathrm{~nm}$; SF: solvent front.

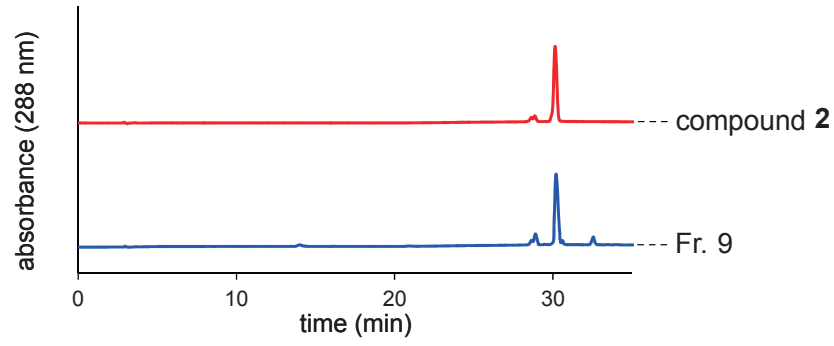

Fig. 5. HPLC chromatograms of standards and fraction 9 after HSCCC separation of the EtOAc extract from the seeds of $S$. marianum. Experimental conditions: Column: Inertsil ODS-4 $(4.6 \times 250 \mathrm{~mm}, 5 \mu \mathrm{m})$; Mobile phase: A/B $0.1 \%$ formic acid/acetonitrile $=75: 25$ (initial) $-75: 25(15 \mathrm{~min})-60: 40(10$ $\mathrm{min})-60: 40$ (10 $\mathrm{min})$; Flow rate: $1.0 \mathrm{~mL} / \mathrm{min}$; Column temperature: $40{ }^{\circ} \mathrm{C}$; Detection: $288 \mathrm{~nm}$.

\section{Conclusions}

The HSCCC was applied to the separation of flavanonol, phenylcoumaran and flavonolignans obtained from the seeds of $S$. marianum. As the result, it was revealed that silydianin and silybin was separated using the HEMWat system ( $3: 7: 4: 6, \mathrm{v} / \mathrm{v})$ with the lower mobile phase. Silybin was also directly separated from the EtOAc extract at high purity. The present HSCCC is useful for the simple separation of silybin because it is known as a bioactive compound.

\section{Acknowledgments}

This work was in part supported by the "Private University Research Branding Project" from MEXT, and also by Nihon University Chairman of the Board of Trustees Grant.

\section{Conflicts of Interest}

The authors declare no conflict of interest.

\section{References}

[1] Sy-Cordero, A.; Graf, T. N.; Nakanishi, Y.; Wani, M. C.; Agarwal, R.; Kroll, D. J.; Oberlies, N. H. Planta Med. 2010, 76, 644-647.

[2] Kim, N. C.; Graf, T. N.; Sparacino, C. M.; Wani, M. C.; Wall, M. E. Org. Biomol. Chem. 2003, 1, 1684-1689.

[3] Alikaridis, F.; Papadakis, D.; Pantelia, K.; Kephalas, T. Fitoterapia 2000, 71, 379-384.

[4] Ahmed, A. A.; Mabry, T. J.; Matlinn, S. A. Phytochemistry 1989, 28, 1751-1753.

[5] AbouZid, S. F.; Chen, S. N.; Pauli, G. F. Ind. Crops. Prod. 2016, 83, 729-737.

[6] Brinda, B. J.; Zhu, H. J.; Markowitz, J. S. J. Chromatogr. B. 2012, 902, 1-9.

[7] Abenavoli, L.; Capasso, R.; Milic, N.; Capasso, F. Phytother. Res. 2010, 24, 1423-1432.

[8] Polyak, S. J.; Morishima, C.; Shuhart, M. C.; Wang, C. C.; Liu, Y.; Lee, D. Y. Gastroenterology 2007, 132, 1925-1936.

[9] Ahmed-Belkacem, A.; Ahnou, N.; Barbotte, L.; Wychowski, C.; Pallier, C.; Brillet, R.; Pohl, R. T.; Pawlotsky, J. M. Gastroenterology 2010, 138, 1112-1122.

[10] Morishima, C.; Shuhart, M. C.; Wang, C. C.; Paschal, D. M.; Apodaca, M. C.; Liu, Y.; Sloan, D. D.; Graf, T. N.; Oberlies, N. H.; Lee, D. Y.; Jerome, K. R.; Polyak, S. J. Gastroenterology 2010, 138, 671-681.

[11] He, Y.; Zhu, S.; Wu, C.; Lu, Y.; Tang, Q. Molecules 2018, 23, 3135.

[12] Zhang, L.; Yue, H. L.; Zhao, X. H.; Li, J.; Shao, Y. J. Chromatogr. Sci. 2015, 53, 860-865.

[13] Liang, Z.; Li, B.; Liang, Y.; Su, Y.; Ito, Y.; J. Liq. Chromatogr. Relat. Technol. 2015, 38, 647-653.

[14] Shinomiya, K.; Sato, K.; Yoshida, K.; Tokura, K.; Maruyama, H.; Yanagidaira, K.; Ito, Y. J. Chromatogr. A 2013, 1322, 74-80.

[15] Shinomiya, K.; Yoshida, K.; Tokura, K.; Tsukidate, E.; Yanagidaira, K.; Ito, Y. Anal. Sci. 2015, 31, 211-218.

[16] Zhu, J. Y.; Cheng, B.; Zheng, Y. J.; Dong, Z.; Lin, S. L.; Tang, G. H.; Gu, Q.; Yin, S. RSC $A d v$. 2015, 5, 12202-12208.

[17] Li, L.; Seeram, N. P. J. Agric. Food Chem. 2010, 58, 11673-11679.

[18] In, S. J.; Seo, K. H.; Song, N. Y.; Lee, D. S.; Kim, Y. C.; Baek, N. I. Arch. Pharm. Res. 2015, 38, 26-34.

[19] Jeon, S. H.; Chun, W.; Choi, Y. J.; Kwon, Y. S. Arch. Pharm. Res. 2008, 31, 978-982.

[20] Friesen, J. B.; Pauli, G. F. J. Liq. Chromatogr. Relat. Technol. 2005, 28, 2777-2806. 